\title{
Visualizing reaction fronts and transport limitations in solid-state Li-S batteries via operando neutron imaging
}

Robert Bradbury ${ }^{\mathrm{a}, \mathrm{b}}$, Georg F. Dewald ${ }^{\mathrm{c}, \mathrm{d}}$, Marvin A. Kraft ${ }^{\mathrm{e}}$, Tobias Arlt ${ }^{\mathrm{a}}$, Nikolay Kardjilov ${ }^{\mathrm{b}}$, Jürgen Janek ${ }^{\mathrm{c}, \mathrm{d}}$ Ingo Manke ${ }^{\mathrm{b}, *}$, Wolfgang G. Zeier ${ }^{\mathrm{e}, \mathrm{f}}$, , Saneyuki Ohno,

${ }^{a}$ Technical University Berlin, Strasse des 17. Juni 135, D-10623 Berlin, Germany

${ }^{b}$ Helmholtz Zentrum Berlin, Hahn Meitner Platz 1, D-14109 Berlin, Germany

'Institute of Physical Chemistry, Justus-Liebig-University Gießen, Heinrich-Buff-Ring 17, D-35392 Gießen, Germany

${ }^{d}$ Center for Materials Research (LaMa), Justus-Liebig-University Gießen, Heinrich-BuffRing 16, D-35392 Gießen, Germany

eInstitute of Inorganic and Analytical Chemistry, University of Münster, Correnstrasse 30, 48149 Muenster, Germany

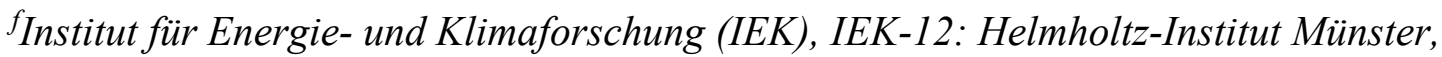
Forschungszentrum Jülich, 48149 Münster, Germany

${ }^{g}$ Department of Applied Chemistry, Graduate School of Engineering, Kyushu University, 744 Motooka, Nishi-ku, 819-0395 Fukuoka, Japan.

Correspondingauthor emails: manke@helmholtz-berlin.de; wzeier@uni-muenster.de; saneyuki.ohno@cstf.kyushu-u.ac.jp 


\begin{abstract}
The exploitation of high-capacity conversion-type materials such as sulfur in solid-state secondary batteries is a dream combination for achieving improved battery safety and high energy density in the push towards a sustainable future. Yet, the exact rate-limiting step, bottlenecking further development of solid-state lithium-sulfur batteries, has not been determined. Here, we directly visualize the spatial distribution of lithium via neutron imaging during operation and show that sluggish macroscopic ion transport within the composite cathode is rate-limiting. Observing a reaction front propagating from the separator side towards the current collector confirms detrimental influences of a low effective ionic conductivity. Furthermore, irreversibly concentrated lithium in the vicinity of the current collector, revealed via state-of-charge-dependent tomography, highlights a hithertooverlooked loss mechanism triggered by sluggish effective ionic transport within a composite cathode. This discovery will be a cornerstone for future research on solid-state batteries, irrespective of the type of active material.
\end{abstract}




\section{Main}

Solid-state batteries offer the potential for higher energy density and increased safety and are therefore currently discussed as an alternative to lithium ion batteries. ${ }^{1,2}$ By combining the concepts of solid-state batteries and of conversion-type lithium-sulfur (Li-S) cells with high theoretical specific energy and natural abundance of sulfur active material, ${ }^{3,4}$ solid-state Li$\mathrm{S}$ batteries have the potential to cause a paradigm shift through simultaneous enhancement of long-term stability and improved battery safety, together with boosted energy density (Wh $\left.\mathrm{kg}^{-1}\right) .{ }^{5}$ Nevertheless, solid-state Li-S batteries do not yet fulfil performance expectations, and further improvement is required. ${ }^{6}$ Electrochemical reactions require a low-impedance supply of ions and electrons but sulfur active materials are ionically and electronically insulating. Hence, for a functional cathode, the sulfur active materials need to be composited with ion and electron conductive additives. These composites provide sufficient triple-phase boundaries where consecutive conversion reactions take place in which the active material can receive or donate ions and electrons. ${ }^{7}$ As a result, a functional sulfur cathode often contains a high interfacial area per unit volume $\left(>10^{4}-10^{6} \mathrm{~cm}^{-1}\right){ }^{8}$

In particular, the rate-limiting step in solid-state Li-S batteries is, at present, not well understood. Despite the very short diffusion length and substantial amounts of triple-phase boundaries achieved after the compositing procedure, slow diffusion of charge carriers in sulfur active materials ${ }^{9}$ and high-impedance charge transfer over the electrolyte-active material interfaces ${ }^{10}$ have often been cited as the rate-limiting steps. ${ }^{11}$ In contrast, the sluggish ion transport within the composite itself, which becomes exponentially worse at high potential, strongly contributes to a high overpotential. ${ }^{8}$ This slow-downed ion transport is not limited to solid-state Li-S batteries and the same transport limitations within the cathode composite are found in intercalation type solid-state batteries. ${ }^{12,13}$ In other words, no matter what cathode active material is used, a low effective ionic conductivity of the cathode composite may be the bottleneck for solid-state battery development. Electrochemical impedance spectroscopy can provide details on the ionic transport. However, a visualization of the overall spatial distribution of transport limitations is essential for determining critical bottlenecks towards high-loading and thick cathode design, as well as fast charging/discharging. Thus, the development of a technique that can monitor cells in 
operando and visualize the distribution of lithium is needed. ${ }^{14}$

Notably, progress in the field of X-ray imaging allowed for the elucidation of solid-state batteries while under operation. ${ }^{15-18}$ However, the identification of lithium is challenging. Here, neutron-based techniques appear promising due to the high neutron attenuation of lithium compared to other elements that comprise the cathode composite. Neutron radiography has been successfully employed in the characterization of batteries with liquid electrolytes. ${ }^{19-21}$ In solid systems, operando neutron diffraction has been conducted to investigate the crystallization of solid electrolytes and lithium transport in garnet-based solid-state cells via depth profiling. ${ }^{22,23}$ To the best of our knowledge, operando neutron analysis has not yet, to date, been performed on solid-state batteries.

In order to perform the operando neutron analysis on the solid-state cell, a cell housing made from aluminium, with a hollow interior was developed, as depicted in Figure 1a. To ensure electrical insulation of the cell, the stack was prepared inside a polyimide tube. The battery components were sandwiched between two stainless steel stamps with O-ring seals to protect the lithium-thiophosphate-based solid-state Li-S battery ( $\mathrm{In} / \mathrm{Li}\left|\mathrm{Li}_{6} \mathrm{PS}_{5} \mathrm{Cl}\right| \mathrm{S} / \mathrm{C} / \mathrm{Li}_{6} \mathrm{PS}_{5} \mathrm{Cl}$ ) from exposure to air and moisture during cycling. A thick cathode configuration delivering an areal capacity of almost $12 \mathrm{mAh} \mathrm{cm}^{-2}$ was employed to better observe changes in the cathode composite layer (see Figure S1 for details of the cell performance).

The neutron beam transmitted through the cell can visualize the components with the level of contrast determined by the degree of neutron absorption (See Table S1). Figure 1b shows a representative 2-D neutron radiograph of the cell before cycling. Darker areas represent the components with low neutron transmission or high absorption. Among the elements comprising the cathode and electrolyte regions, lithium possesses the highest neutron absorption coefficient, ${ }^{24}$ thus the contrast changes are primarily due to the variations in lithium concentration. In the anode, the presence of indium should be noted since it has an even higher neutron absorption coefficient than lithium, ${ }^{24}$ which is reflected in Figure $1 \mathrm{~b}$ where the attenuation of the neutron beam is much greater in the anode. Overall, since indium remains immobile throughout the electrochemical cycling, mobile lithium can be considered to be responsible for all changes in neutron attenuation. The neutron attenuation measured 
in the pristine (as-prepared) and fully discharged cells show distinct differences (Figure 1c). These are most evident in the cathode, where the change from the pristine to the fully discharged state is significant. It is consistent with what is occurring in the cell during discharge - there is a net loss in lithium from the anode and a net gain at the cathode, while the solid electrolyte separator exhibits no overall change.
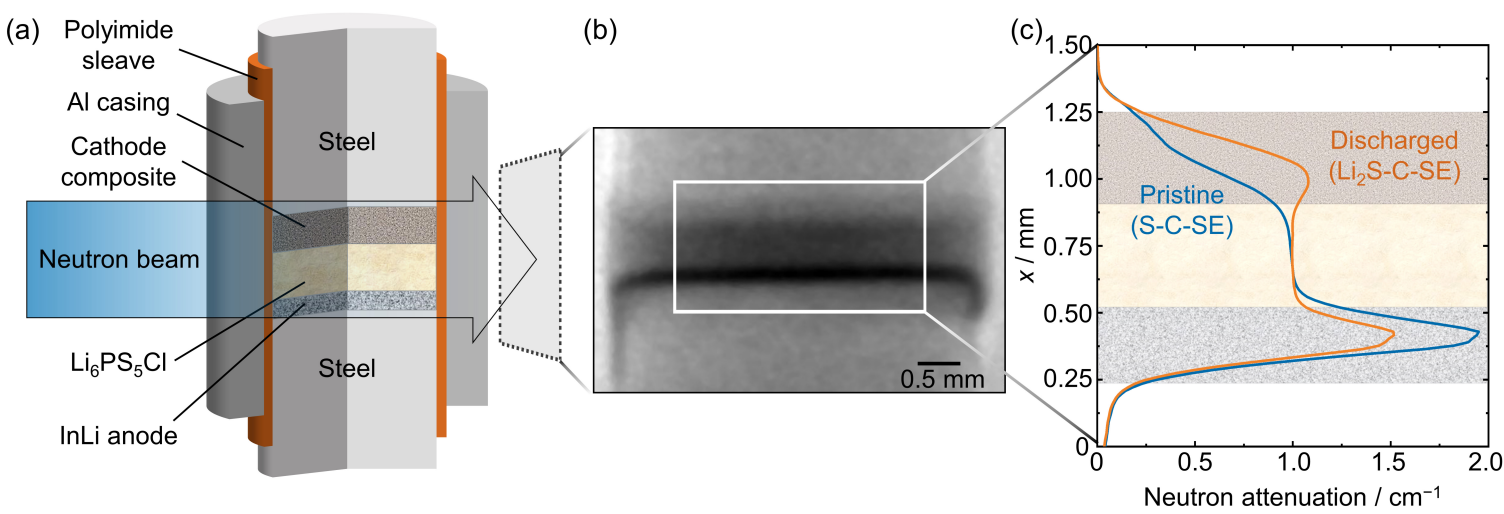

Figure 1: Cell design, representative neutron radiography, and neutron attenuation. (a) Schematic representation of the experimental setup for neutron imaging. A custom-made cell optimized for neutron imaging was employed. (b) The element-specific neutron attenuation allows clear differentiation of the cell components from the steel and aluminium in the neutron radiogram. (c) The position-dependent neutron attenuation quantified within the white box in (b) before and after the initial discharge visualizes the variation in the distribution of the Li concentration.

Whereas in situ neutron tomography offers the opportunity to quantify the lithium distribution through the cathode in 3-D at specific charge states, 2-D radiography, conducted operando, can visualize the changes in lithium distribution through the cathode composite during cathode lithiation/delitiation. The solid-state Li-S cell was cycled at $1.13 \mathrm{~mA} \mathrm{~cm}^{-1}$ while in the neutron beam (Figure 2a). Throughout the initial discharge images were recorded, each with an exposure time of $10 \mathrm{~s}$ and a pixel size of $13 \mu \mathrm{m}$. After processing the images to account for the background and normalizing them to the initial pristine state, what is now represented is the change in the number of transmitted neutrons relative to the initial 
state. However, since the images are a 2-D representation of a cylindrical cell, the path taken by the neutrons $\left(l_{\mathrm{p}}\right)$ is not constant across the image. This can be resolved by using the BeerLambert law (see Supplementary Information) to convert neutron transmission to attenuation. Neutron attenuation is dependent on $l_{\mathrm{p}}$ and has units of $\mathrm{cm}^{-1}$. To do this, it is necessary to consider each pixel in the image individually and, working out from the centre where $l_{\mathrm{p}}$ is the cell diameter, the path length for each can be calculated using simple trigonometry. Compiling the images together in sequence, it becomes clear how the attenuation changes with time (see Supplementary Video 1) with a brighter area, reflecting the local presence of more lithium. In order to focus attention on the cathode composite only a region between a point in the solid electrolyte separator denoted by $d_{0}$, and a point in the steel current collector, denoted by $d_{\max }$ is displayed. Figure S2 displays the attenuation change for the cathode at $10 \%$ intervals in the depth of discharge (DoD).

If we consider how each pixel changes with time, taking the derivative offers a clear picture of where the lithium is distributed in the cathode composite and when it arrives, as shown in Supplementary Video 2. The heatmap in Figure $2 \mathrm{~b}$ highlighting the median rate of neutron attenuation change ( $\triangle \mathrm{Attn}_{\mathrm{Neu}}$ ) shows that there is a distribution through the cathode of the rate at which the attenuation changes. The maximum rate of attenuation change progresses through the cathode with time. Figure $2 \mathrm{c}$ shows the propagation of this "reaction front" through the cathode as a function of $\mathrm{DoD}$ (the full cathode width images of the rate of change of attenuation are displayed in Figure S3 at 10\% intervals of DoD). The shift in the maximum rate of change is highlighted in Figure $2 \mathrm{~d}$ whereby the median rate of attenuation change is displayed as a function of $d$ for $10 \%$ DoD intervals. The edges of the cell deform slightly due to the soft polyimide sleeve used for cell insulation and it broadened the peak shape. This occurs around the full circumference of the cell but is only clearly visible in Figure $2 b$ for the edges of the cell perpendicular to orientation of the neutron beam. This, and the limitation of the instrument resolution are responsible for the broadening of the peak shape as well as spill-over into the region of the steel current collector when $d>d_{\text {Cathode|CC }}$ and the separator when $d<d_{\mathrm{SE} \mid \text { Cathode. }}$ Variation in the rate of attenuation change is to be expected across the cell throughout electrochemical cycling but an area in the cathode composite will experience the greatest rate of change when the lithium front first arrives. Despite the known 
volume expansion of the cathode composite upon discharging, ${ }^{25}$ it is clear that this reaction front propagates from the separator-side towards the current collector as the cell discharges.

(a)

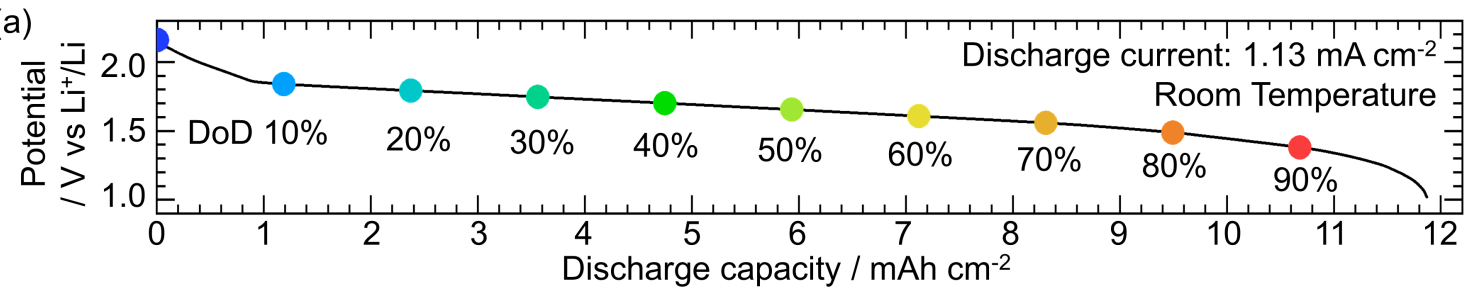

(b)

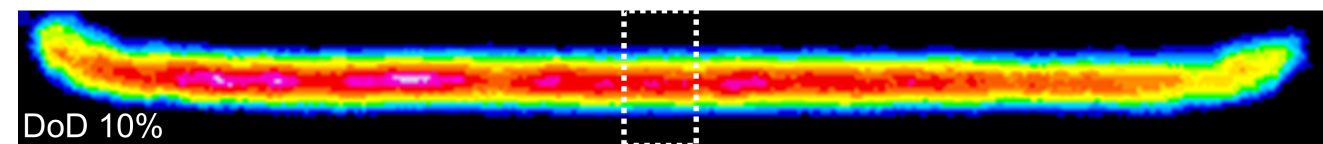

(c)

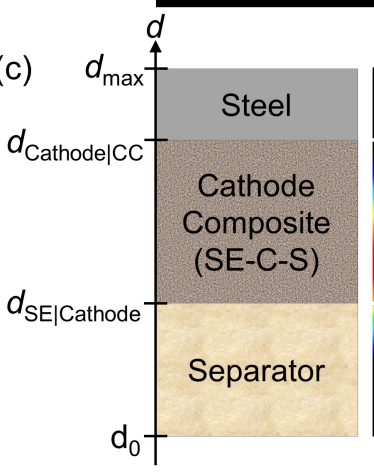

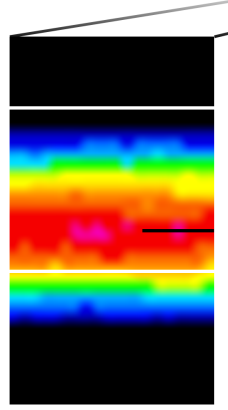

DoD $10 \%$

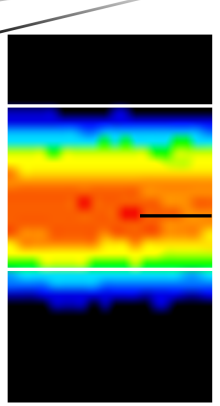

DoD $30 \%$

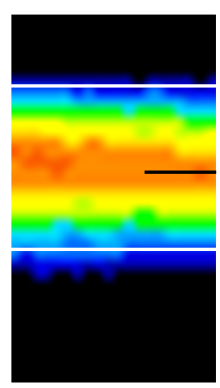

DoD $50 \%$

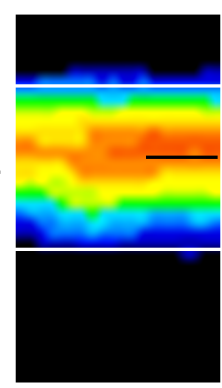

DoD $70 \%$

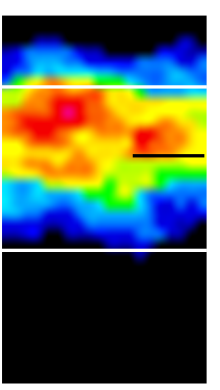

DoD $90 \%$

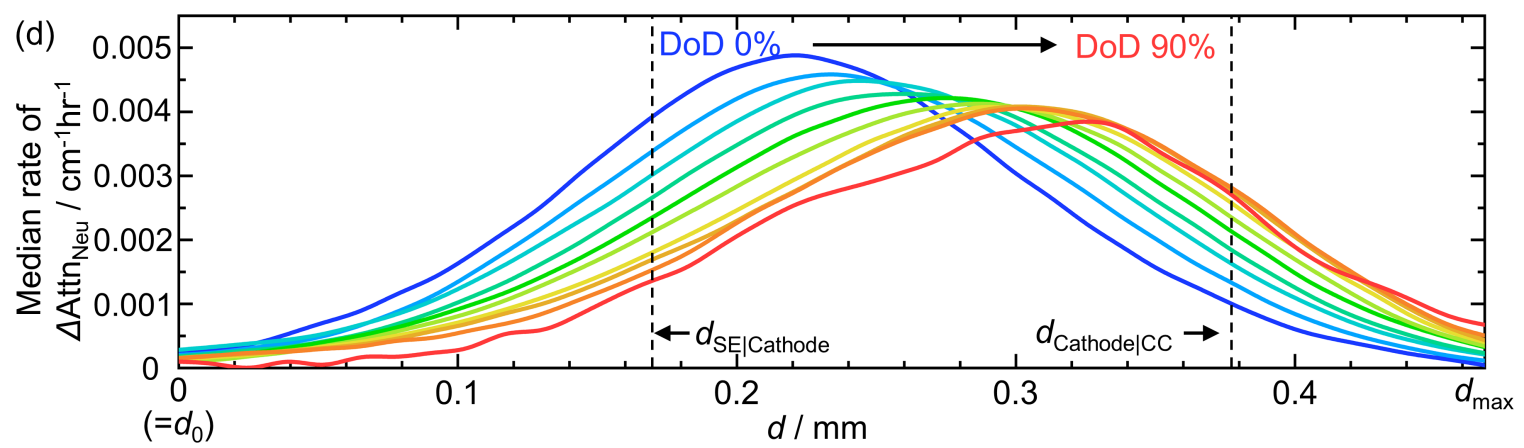

Figure 2: Dynamics of lithium distribution visualized by operando neutron radiography.

(a) Voltage decrease plotted as a function of discharge capacity. Each data point represents the degree of discharge (DoD). (b) Neutron radiography image of the cathode composite at $10 \%$ DOD. The colour scale represents rate of change in neutron attenuation. In the vertical direction the image ranges from $d_{0}$ to $d_{m a x}$. (c) Progression of the point of maximum rate of attenuation change (reaction front) towards higher $d$ as DoD increases. The region displayed for each DoD is taken from the dotted area shown in (b) (for 10\% DoD). The white lines 
represent the cathode interfaces with the steel current collector (upper) and the solid electrolyte separator (lower), which were determined based on the data at $0 \%$ DOD in combination with the 3-D tomography data. The black likes represent to the position giving the maxima median rate of attenuation change in (d). (d) Median rate of attenuation change as a function of $d$. The dotted lines represent the interfaces as denoted by the white lines in (c).

While operando neutron radiography provides information regarding the dynamics of lithium transport through the cathode composite during cycling, this insight is limited by the two-dimensional nature of the technique, describing the lithium distribution only in terms of $d$. To acquire information on the homo- or heterogeneous distribution in the cathode area for a given $d$, a different neutron imaging technique, neutron tomography, is required. Although the information on the dynamics will be lost since tomography requires a series of images to be recorded as the sample is rotated, these images, taken in situ, can be reconstructed to create a 3-D representation of the cell (see Figure S4 showing the pristine, discharged, and recharged states).

Through subtraction of the pristine state from subsequently measured tomograms of fully discharged and re-charged cells, it is now possible to directly visualize changes to the lithium distribution across the whole volume. Figure 3a shows two 3-D representations of the cell, one in a discharged (top) and the other in a charged state (middle). Each tomogram has been normalized to the pristine state to show only the changes in the cell effected during cycling. The anode shows the negative attenuation change and the separator region has zero net change in total lithium, similar to the steel stamp below the anode and above the cathode. The separator region is denoted in grey. The cathode, which is the focus of our in-depth analysis, displays a notable difference between the two charge states. This is clearly demonstrated in the third representation (bottom) which shows the difference between the two charge states (the anode difference is less noticeable due to percentage changes to its overall attenuation being much less than the cathode). Figures $3 \mathrm{~b}$ and $\mathrm{c}$ display the attenuation change in $39 \mu \mathrm{m}$ thick slices from $d_{0}$ to $d_{\max }$ for the fully discharged and re- 
charged states, respectively. The difference in attenuation between the discharged and charged states represents the mobile lithium that is stripped from the cathode upon charging. The origin of the non-zero rate of attenuation change observed beyond the cathode|current collector interface in Figure 2d, and attributed to the deformation of the cathode outer edge, is also seen in the final three images in both Figures $3 b$ and $c$. If we take each image from the series and sum the attenuation change of all voxels within the cell area, the differing behaviour across the depth of the cathode composite becomes even clearer (Figure 3d). The discharged state (orange plot) represents the lithium that has accumulated in the cathode upon lithiation of $\mathrm{S}$ to form $\mathrm{Li}_{2} \mathrm{~S}$ during initial discharge. It is evident from both Figure $3 \mathrm{c}$ and $3 \mathrm{~d}$ that the lithium is heterogeneously distributed throughout the cathode. The attenuation from the re-charged state (blue plot) represents lithium that is trapped within the cathode composite, in the form of $\mathrm{Li}_{2} \mathrm{~S}$ that cannot be converted back to $\mathrm{S}$. This trapped lithium is observed across the cathode depth in Figure $3 \mathrm{~d}$ but is notably greater at higher $d$, the current collector side of the cathode composite. The mobile lithium that is not trapped in the cathode and which is transported back towards the anode when the cell is recharged can be quantified as the difference between the lithium present in the cathode in the discharged state and that which remains after the cell has been charged. In Figure $3 \mathrm{~d}$ (green plot) this lithium can be shown to have been located at, and lost from the solid electrolyte separator side of the cathode composite, at lower $d$. 
(a) Discharged

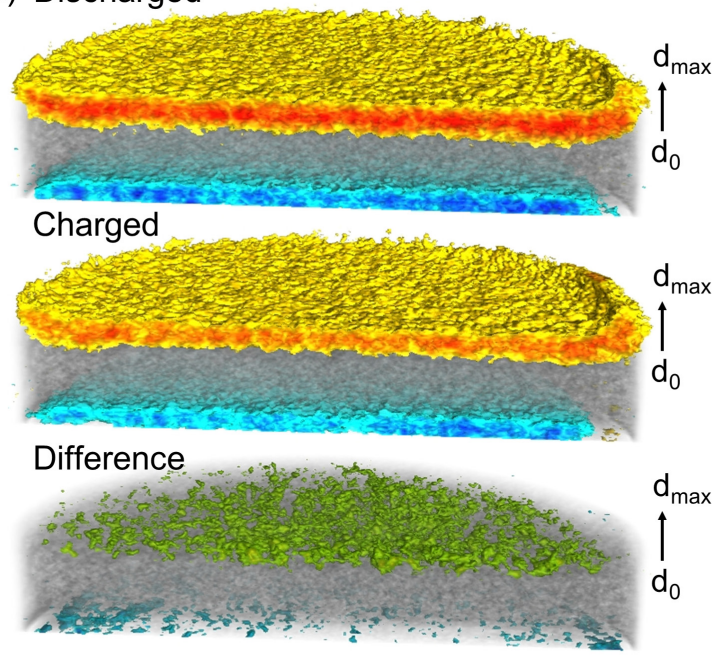

(d)

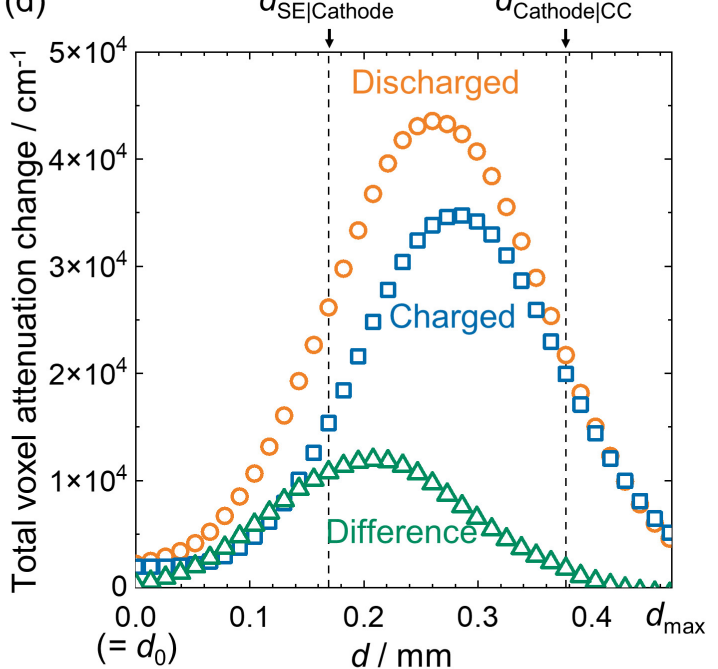

(b)

(c)

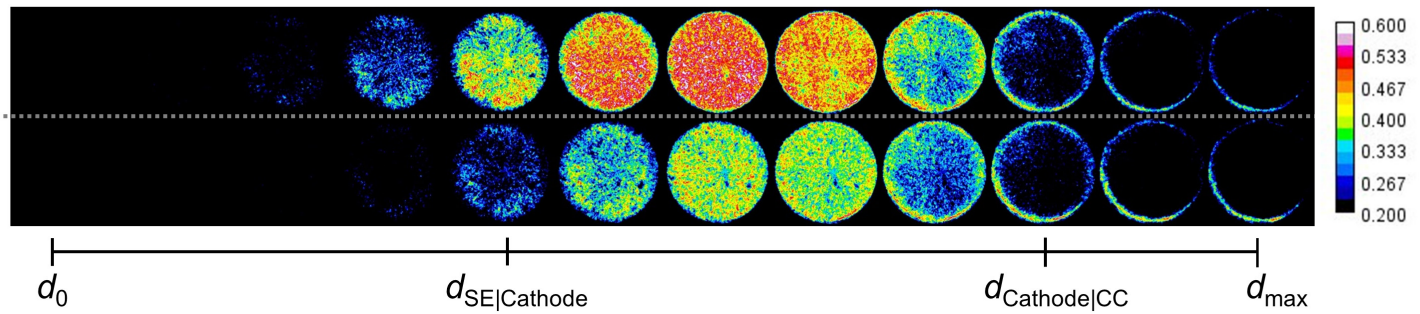

Figure 3: In-situ neutron tomography on the discharged and charged solid-state sulfur cathode. (a) 3-D tomography images of the discharged (top) and recharged state (middle), normalised to the initial state to emphasize changes in the cell, along with the difference between them (bottom) that shows the location of the mobile lithium. (b) Median neutron attenuation change through the cathode composite in the discharged state normalized to the pristine state. The images range from $d_{0}$ at the edge of the solid electrolyte region to $d_{\max }$ on the current collector side of the cathode composite. (c) Same as (b) but for the re-charged state. The colour bar in both represents the range of attenuation change above that of the background. (d) Total neutron attenuation change for $13 \mu \mathrm{m}$ thick slices through the cathode composite for the discharged (orange) and re-charged states (blue). The latter represents the lithium trapped in the cathode. The difference between the two charged states represents the mobile lithium (green). 
Assuming that the cathode composite is homogeneous and has substantially high interfacial area density, we expand a conventional "one-dimensional porous electrode theory", also known as the Newman model, ${ }^{26}$ to solid-state sulfur cathodes. The model was originally developed to describe the distribution of the reaction current within an electrode consisting of a porous matrix of a reactive material and a liquid electrolyte that has penetrated into voids in the porous material. A dimensionless reaction rate describes a distribution of the reaction current in a thickness direction of the cathode composite, which corresponds to the rate of attenuation change observed in this work. A detailed description can be found in the Supplementary Information.

When the effective ionic and electronic conductivities ( $\sigma_{\text {ion }}^{\text {eff }}$ and $\sigma_{\mathrm{e}}^{\text {eff }}$ ) of a cathode composite are sufficiently fast with respect to the applied current and thickness, the distribution of reaction current becomes uniform as shown in Figure $4 \mathrm{~b}$. With fast supply of ions and electrons, charge transfer and lithium diffusion within the active material particle will remain as potential rate-limiting steps. In contrast, when the effective transport in the composite is insufficient, the reaction current distribution becomes non-uniform, as shown in Figure $4 \mathrm{c}$ and $\mathrm{d}$, and the overvoltage due to the sluggish transport within the electrolyte in the composite becomes non-negligible. It is notable that with $\sigma_{\mathrm{e}}^{\text {eff }} \gg \sigma_{\mathrm{ion}}^{\text {eff }}$, the model predicts a non-uniform reaction rate with formation of a reaction front propagating from the separator-layer side toward the current collector (Figure 4c). ${ }^{27}$ With comparable $\sigma_{\mathrm{e}}^{\text {eff }}$ and $\sigma_{\text {ion }}^{\text {eff }}$, another reaction front from the current collector side will form, and, lastly, the reaction front on the separator side will diminish when $\sigma_{\text {ion }}^{\text {eff }} \gg \sigma_{\mathrm{e}}^{\text {eff }}$ (Figure 4d). Considering that the here-employed composite possesses effective ionic and electronic conductivities of $\left(4.5 \cdot 10^{-3} \pm 0.5\right) \mathrm{mS} \mathrm{cm}{ }^{-1}$ and $(20 \pm 4) \mathrm{mS} \mathrm{cm} \mathrm{cm}^{-1}$, respectively (see Supplementary Information), the experimental results of operando neutron measurements, visualizing the reaction front from the separator side toward the current collector, agree well with theoretical prediction. The degree of non-uniformity is parameterized by $\delta$, which is a function of the applied current, the cathode thickness, and effective conductivities. The pristine cell, visualized here via neutron measurements, would correspond to $\delta \sim 2 \times 10^{2}$, predicting a severely uneven distribution of reaction current, as observed in the experiment. The details 
of calculation are shown in the Supplementary Information.

The presence of a reaction front propagating toward the current collector further implies an overlooked capacity loss mechanism in a thick composite cathode. A reaction rate distribution should be identical for both anodic and cathodic currents within the model, as long as the given parameters are unchanged (see Figure $4 \mathrm{c}$ and d). However, in practice, an effective ionic conductivity of the cathode composite experiences a substantial decrease upon delithiation above $2 \mathrm{~V}$ vs. In/InLi (2.62 vs. $\left.\mathrm{Li}^{+} / \mathrm{Li}\right),{ }^{8,28}$ leading to a gradient in lithium concentration in the thickness direction of the cathode composite. During the propagation of the reaction front from the separator side, the effective ionic conductivity of the composite decreases, and further delithiation from the current collector sides will be hindered. Consequently, the lithium concentration gradient in the thickness direction remains after the re-charging process, as observed in the in situ neutron tomography. This most likely accounts for the commonly observed asymmetric overpotential between discharge and charge after cycling. ${ }^{8}$ Exemplary asymmetric potential profiles of a solid-state Li-S battery with the same cathode composite are shown in Figure S5. Irrespective of the nature of active materials, the slow ionic transport in composites has been shown in intercalation type solid-state batteries as well, suggesting that the here-found phenomena will also play a role in other types of solid-state batteries.

Therefore, a non-uniform reaction in the thickness direction caused by sluggish ion transport of the cathode composite highlights the necessity of (1) a processing procedure without sacrificing fast ion transport, (2) a design principle of cathode architecture boosting the effective ionic conductivity, and (3) further development of solid electrolytes possessing both high ionic conductivity and electrochemical stability. 


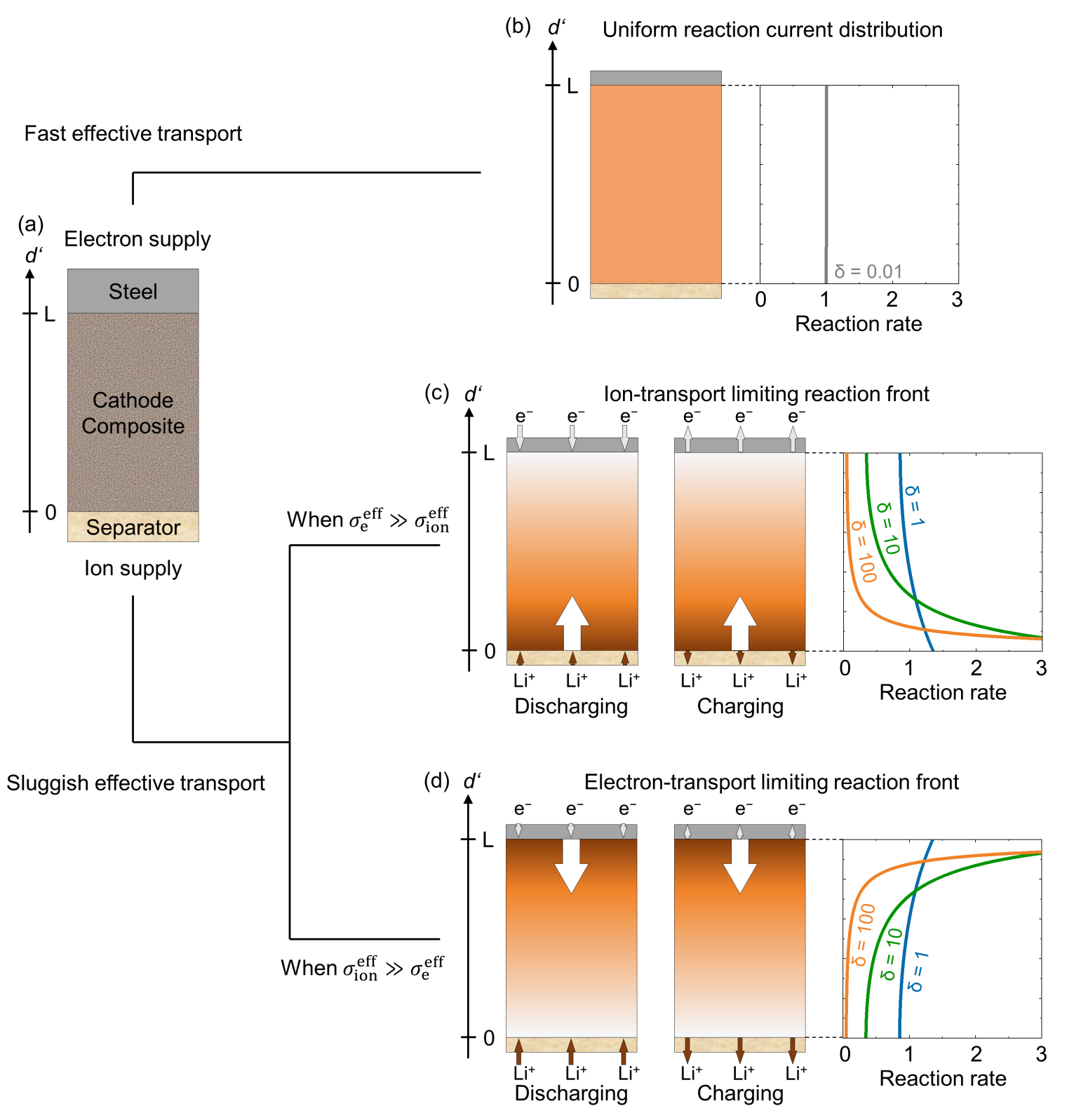

Figure 4: Extension of a porous electrode theory to solid-state sulfur cathodes. (a) A schematic of the cathode composite with a thickness of $L$ and an applied current of I. $A \delta$ parameter is given by $\delta \propto L|I|\left(\frac{1}{\sigma_{\mathrm{ion}}^{\text {eff }}}+\frac{1}{\sigma_{\mathrm{e}}^{\text {eff }}}\right)$. (b) Porous electrode theory predicts a uniform reaction rate distribution with fast effective carrier transport. (c) Sluggish ion transport within the composite leads to a non-uniform reaction rate, forming a reaction front propagating from the electrolyte-layer side toward the current collector. (d) With low 
effective electronic conductivity, the reaction front propagates from the opposite side. The reaction rate distribution becomes less steep with lower $\delta$ by increasing rate-limiting transport.

\section{Conclusions}

Operando neutron radiography and in situ neutron tomography have successfully been demonstrated and elucidate the transport limitations in a solid-state sulfur cathode composite. 2-D radiographs reveal a reaction front, propagating from the separator-layer side toward the current collector upon the initial discharge. 3-D tomography visualizes residual lithium concentrated in the vicinity of the current collector after re-charging. Extending the porous electrode theory for solid-state batteries corroborates that the sluggish effective lithium-ion transport in composites is rate-limiting and leads to a non-uniform reaction front.

This work peeks into the lithium dynamics inside composite cathodes in solid-state batteries, showing that limitations exist in cathode composites due to slow ionic transport. Having observed this reaction front urges further work in cathode composite design of solid-state batteries in general because high loading and thick cathode composites, as well as fast charging/discharging, are needed for a realistic solid-state battery implementation.

\section{Acknowledgments}

This research was supported by the Federal Ministry of Education and Research (BMBF) within the project LISZUBA under grant numbers 03XP0115A and 03XP0115C. SO gratefully acknowledges the Alexander von Humboldt Foundation for partial financial support through a Postdoctoral Fellowship.

\section{Author contributions}

IM, WGZ, and SO designed the project toward the transport limitation. RB, GD, and SO designed the operando cell. GD and SO synthesized materials, prepared the cathode composites, and assembled the cells. RB, GD, TA, and MK performed tomography. RB analyzed the data. RB, NK, and SO interpreted the data. RB and SO prepared the initial draft of the work. All authors gave comments and edited the manuscript. 


\section{Supplementary information}

Supplementary Sections S1-S7 and Figures S1-S5.

\section{Supplementary Video 1}

Progression of the neutron attenuation change over time.

\section{Supplementary Video 2}

Median rate of neutron attenuation change showing a propagation of the reaction front within the cathode from the separator side toward the current collector.

\section{Supplementary Video 3}

State-of-charge-dependent in situ neutron tomography performed at the pristine, discharged, and recharged states.

\section{References}

1. Janek, J. \& Zeier, W. G. A solid future for battery development. Nat. Energy 1, 16141 (2016).

2. Randau, S. et al. Benchmarking the performance of all-solid-state lithium batteries. Nat. Energy 5, 259-270 (2020).

3. Herbert, D. \& Ulam, J. United States Patent Office l 3,043,896 ELECTRIC DRY CELLS AND STORAGE BATTERES. (1958).

4. Ji, X., Lee, K. T. \& Nazar, L. F. A highly ordered nanostructured carbon-sulphur cathode for lithium-sulphur batteries. Nat. Mater. 8, 500-506 (2009).

5. Yang, X., Luo, J. \& Sun, X. Towards high-performance solid-state Li-S batteries: From fundamental understanding to engineering design. Chem. Soc. Rev. 49, 21402195 (2020).

6. Ohno, S. \& Zeier, W. G. Toward Practical Solid-State Lithium-Sulfur Batteries: Challenges and Perspectives. Accounts Mater. Res. accountsmr.1c00116 (2021) doi:10.1021/accountsmr.1c00116. 
7. Dewald, G. F., Ohno, S., Hering, J. G. C., Janek, J. \& Zeier, W. G. Analysis of Charge Carrier Transport Toward Optimized Cathode Composites for All-Solid-State Li-S Batteries. Batter. Supercaps 4, 183-194 (2021).

8. Ohno, S., Rosenbach, C., Dewald, G. F., Janek, J. \& Zeier, W. G. Linking Solid Electrolyte Degradation to Charge Carrier Transport in the Thiophosphate-Based Composite Cathode toward Solid-State Lithium-Sulfur Batteries. Adv. Funct. Mater. 31, 2010620 (2021).

9. Lorger, S., Usiskin, R. E. \& Maier, J. Transport and Charge Carrier Chemistry in Lithium Sulfide. Adv. Funct. Mater. 29, 1807688 (2019).

10. Yu, C. et al. Accessing the bottleneck in all-solid state batteries, lithium-ion transport over the solid-electrolyte-electrode interface. Nat. Commun. 8, (2017).

11. Fan, B. et al. Electrochemical processes in all-solid-state Li-S batteries studied by electrochemical impedance spectroscopy. Solid State Ionics 368, 115680 (2021).

12. Minnmann, P., Quillman, L., Burkhardt, S., Richter, F. H. \& Janek, J. Editors' Choice-Quantifying the Impact of Charge Transport Bottlenecks in Composite Cathodes of All-Solid-State Batteries. J. Electrochem. Soc. 168, 040537 (2021).

13. Bielefeld, A., Weber, D. A. \& Janek, J. Modeling Effective Ionic Conductivity and Binder Influence in Composite Cathodes for All-Solid-State Batteries. ACS Appl. Mater. Interfaces 12, 12821-12833 (2020).

14. Strauss, F. et al. Influence of electronically conductive additives on the cycling performance of argyrodite-based all-solid-state batteries. RSC Adv. 10, 1114-1119 (2019).

15. Lewis, J. A. et al. Linking void and interphase evolution to electrochemistry in solidstate batteries using operando X-ray tomography. Nat. Mater. 2021204 20, 503-510 (2021).

16. Dixit, M. B., Park, J.-S., Kenesei, P., Almer, J. \& Hatzell, K. B. Status and prospect of in situ and operando characterization of solid-state batteries. Energy Environ. Sci. 14, 4672-4711 (2021).

17. Dixit, M. B. et al. Nanoscale Mapping of Extrinsic Interfaces in Hybrid Solid Electrolytes. Joule 4, 207-221 (2020). 
18. Finegan, D. P. et al. In-operando high-speed tomography of lithium-ion batteries during thermal runaway. Nat. Commun. 201561 6, 1-10 (2015).

19. Sun, F. et al. Complementary X-ray and neutron radiography study of the initial lithiation process in lithium-ion batteries containing silicon electrodes. Appl. Surf. Sci. 399, 359-366 (2017).

20. Goers, D. et al. In situ neutron radiography of lithium-ion batteries: The gas evolution on graphite electrodes during the charging. J. Power Sources 130, 221-226 (2004).

21. Lanz, M., Lehmann, E., Imhof, R., Exnar, I. \& Novák, P. In situ neutron radiography of lithium-ion batteries during charge/discharge cycling. J. Power Sources 101, 177$181(2001)$.

22. Wang, C. et al. In Situ Neutron Depth Profiling of Lithium Metal-Garnet Interfaces for Solid State Batteries. J. Am. Chem. Soc. 139, 14257-14264 (2017).

23. Rao, R. P., Sharma, N., Peterson, V. K. \& Adams, S. Formation and conductivity studies of lithium argyrodite solid electrolytes using in-situ neutron diffraction. Solid State Ionics 230, 72-76 (2013).

24. Sears, V. F. Neutron scattering lengths and cross sections. Neutron News 3, 26-37 (1992).

25. Ohno, S. et al. Observation of Chemomechanical Failure and the Influence of Cutoff Potentials in All-Solid-State Li-S Batteries. Chem. Mater. 31, 2930-2940 (2019).

26. Newman, J. S. \& Tobias, C. W. Theoretical Analysis of Current Distribution in Porous Electrodes. J. Electrochem. Soc. 109, 1183 (1962).

27. Neumann, A. et al. Analysis of Interfacial Effects in All-Solid-State Batteries with Thiophosphate Solid Electrolytes. ACS Appl. Mater. Interfaces 12, 9277-9291 (2020).

28. Dewald, G. F. et al. Experimental assessment of the practical oxidative stability of lithium thiophosphate solid electrolytes. Chem. Mater. 31, 8328-8337 (2019). 